\title{
Early Introduction of multi-allergen mixture for prevention of food allergy
}

A. Zoe Quake, Taryn Audrey Liu, Rachel D’Souza, Katherine G. Jackson, Margaret A. Woch, Afua Tetteh, Vanitha Sampath, Kari C. Nadeau, Sayantani Sindher, R. Sharon Chinthrajah, Shu Cao.

Sean N. Parker Center for Allergy and Asthma Research at Stanford University, Division of Pulmonary, Allergy and Critical Care Medicine, Dept of Medicine, Stanford, CA, USA.

Corresponding author: Shu Cao, MSc, Sean N. Parker Center for Allergy and Asthma Research at Stanford University Clinical Research, 240 Pasteur Dr. BMI Rm.1755, Palo Alto, CA 94304, USA. E-mail: shucao@stanford.edu. 


\section{ABSTRACT}

The incidence and prevalence of food allergy (FA) are increasing. While several studies have established the safety and efficacy of early introduction of single allergens in infants for the prevention of FA, the exact dose, frequency, and number of allergens that can be safely introduced to infants particularly in those at high or low risk of atopy are still unclear. This 1year study evaluated the safety of early introduction of single foods (milk, egg, or peanut), vs. two foods (milk/egg, egg/peanut, milk/peanut), vs. multiple foods (milk/egg/peanut/cashew/almond/shrimp/walnut/wheat/fish/soy at low, medium, or high doses) vs no early introduction in infants between 4-6 months of age. At the end of the study, they were evaluated for plasma biomarkers associated with food reactivity with standardized blood tests. Two to four years after the start of the study, participants were evaluated by standardized food challenges. The serving sizes for the single, double, and low dose mixtures were $300 \mathrm{mg}$ total protein per day. The serving sizes for the medium and high dose mixture was $900 \mathrm{mg}$ and 3000 mg total protein, respectively. Equal parts of each protein were used for double or mixture foods. All infants were breastfed until at least 6 months of age. Results demonstrate that infants at either high or low risk for atopy were able to tolerate early introduction of multiple allergenic foods with no increases in any safety issues, including eczema, FA, or food protein induced enterocolitis. The mixtures of foods at either low, medium, or high doses demonstrated trends for improvement in food challenge reactivity and plasma biomarkers compared to single and double food introductions. The results of this study suggest that early introduction of foods, particularly simultaneous mixtures of many allergenic foods is efficacious for preventing FA and can occur safely. 


\section{KEYWORDS: multi-allergen, early introduction, food allergy, prevention, safety, efficacy}

\section{INTRODUCTION}

The incidence and prevalence of food allergy (FA) have increased significantly in recent years (1-3). Today, an estimated $7.6 \%$ of U.S. children and nearly $11 \%$ of adults have reported FAs to at least one food $(4,5)$. Reactions can be mild, moderate, severe, or life-threatening with clinical symptoms varying considerably between individuals and over the lifetime (6). Although a number of foods are known to be allergenic, cow's milk, hen's egg, peanuts, soy, wheat, tree nuts, fish, and shellfish account for $90 \%$ of all FAs (7). Accidental ingestion rates for peanut, egg, and milk range from 14 to $33 \%$ (8), 19-50\%, and 17-36\%, respectively.(9)

There is an urgent and unmet need to prevent and treat FAs. Despite limited data, older guidelines recommended delaying exposure to cow's milk until 12 months, hen's egg until 24 months, and peanut, tree nut, and fish until 3 years (10) to prevent FA. In 2015, results of a large randomized controlled study, the Learning Early About Peanut (LEAP) study challenged these early guidelines and concluded that early introduction of peanuts significantly reduced the likelihood of developing peanut allergy and conversely, delayed introduction significantly increased the likelihood of developing peanut allergy in infants. The study also indicated that delayed introduction may lead to impaired immune responses (11). The benefits of early introduction of allergenic foods for prevention of FA are now supported by a number of randomized controlled trials, particularly for peanut, egg and milk. A meta-analysis found strong evidence of positive benefits of early introduction of allergenic foods.(12)

Diet diversity in infants has also been found to play a role in preventing FA. In 2014, the PASTURE study found that increased diversity of complementary foods introduced in the first 
year of life was inversely associated with doctor-diagnosed FA up to 6 years of age, food sensitization, and increased expression of a T regulatory cell marker.(13) Diet diversity was also evaluated in a large prospective study using standardized questionnaires. The study showed that the introduction of each additional food at 6 and 12 months of age reduced by $10.8 \%$ and $33.2 \%$ respectively, the odds of developing FA over the first 10 years of life (14). Systemic reviews suggest that diet diversity in infancy may be associated with reduced allergy outcomes (including FA). $(15,16)$

Among children with FA, up to $40 \%$ of children are allergic to multiple foods (5). This suggest that need to include multiple allergenic foods into the diets of these infants for prevention of FA as research shows that it is allergen-specific (17). At the current time, only a few studies have looked at the introduction of multiple allergenic foods for prevention of FA. In the Enquiring About Tolerance (EAT) study, infants were randomized at 3 months and sequentially introduced to 6 allergenic foods (including cow's milk, peanut, hard-boiled egg, sesame, cod and wheat) vs. to a standard group where infants avoided allergenic foods. Per protocol analysis indicated that the early introduction group had a $67 \%$ relative risk reduction of any FA compared to the standard group (18). While the EAT study showed that it was safe and effective to introduce multiple allergens, the amounts of allergens used were impractical as per the USDA guidelines for daily caloric intake in infants $(528,619$, and 806 calories daily for infants 4, 6, and 12 months respectively). (19) Infants in the EAT study were asked to consume the equivalent of $4 \mathrm{~g}$ of each allergenic protein per week which amounted to 2 small 40 - to $60-\mathrm{g}$ 
portions of cow's milk yogurt, 3 rounded teaspoons of peanut butter, 1 small hard-boiled egg, 3 rounded teaspoons of sesame paste, $25 \mathrm{~g}$ of whitefish, and 2 wheat-based cereal biscuits. There were 10 participants whose families reported food protein induced enterocolitis syndrome (FPIES) like reactions, seven in the early-introduction group and three in the standardintroduction group. The difference between the two groups was not statistically significant $(\mathrm{p}=0.34)$. Holl et al. conducted a blinded randomized placebo-controlled study to determine whether early introduction of multiple allergens simultaneously is acceptable to caregivers and tolerable to any healthy infant. In this study, the 16 most common allergenic foods (peanut, soy, almond, cashew, hazelnut, pecan, pistachio, walnut, wheat, oat, milk, egg, cod, shrimp, salmon, and sesame) were premixed and fed to infants between the ages of 5-11 months, for a total of $480 \mathrm{mg}$ of proteins per day for 28 days. This study had a high completion rate indicating acceptability and showed no significant difference in safety between the active vs placebo group $(p=0.76)$. There were no increases in adverse events and there were no increases in reported IgEtype allergic reactions in the active vs. placebo group (20). In fact, the placebo group had allergic reactions likely due to delayed introduction of allergenic foods.

As evidence increasingly suggested that diet diversity and early intervention may decrease FA $(11,21)$, infant feeding guidelines were revised and currently recommend full introduction of diverse allergenic foods at 4 to 6 months (22-24). However, the early and consistent introduction of not just single allergens, but multiple allergens can be difficult to 
administer in infants. Further research on the number of allergens, amount of allergenic proteins, and length of time allergens need to be consumed is needed to optimize tolerability while providing a convenient and practical method for early introduction of proteins to infants. Therefore, in the current study, we conducted a 1-year-feeding randomized clinical trial to evaluate safety of simultaneously introducing a multi-allergen mix vs. double mix vs. single foods vs no early introduction in infants with high and low risk of allergy. In addition to clinical evaluations of ingestion tolerance via oral food challenges, biomarkers were also measured (allergen-specific $\mathrm{IgE}$ and $\left.\mathrm{IgG}_{4}\right)$. 


\section{MATERIALS AND METHODS}

The study was conducted at the Sean N. Parker Center for Allergy and Asthma Research at Stanford University. Both high risk infants and low risk infants were enrolled and stratified 1:1. High risk was defined as one first degree relative with FA/atopic dermatitis or two first degree relatives with atopic disease.(25). Exclusion criteria included infants with chronic diseases or known genetic diseases or with known FA. The study was conducted with IRB approval from Stanford University between June 2015 and January 2018 and infants between 2 months and 12 months of age were enrolled (NCT04828603). Ethical approval (8629) was obtained on April 15, 2015. Participants were randomized (but not blinded) equally: singles (egg, milk, or peanut; $\mathrm{n}=15$ each), doubles in equal parts by mg protein (peanut plus milk, peanut plus egg, or milk plus egg, $\mathrm{n}=15$ each), or a 10-allergen mixture (almonds, cashew, egg, hazelnut, milk, peanut, salmon, shrimp, walnut, wheat) and an age- and sex-matched control group $(n=45)$ which avoided all potentially allergenic foods for the first year of the study. The serving sizes for the 10-allergen mixture were low, medium, or high (i.e., $300 \mathrm{mg}$ per day, $\mathrm{n}=1 ; 900 \mathrm{mg}$ per day, $\mathrm{n}=15 ; 3000 \mathrm{mg}$ per day, $n=15$, in equal 1:1 parts of the 10 proteins, respectively). Participants were fed the first serving in the clinic and observed for 2-hours post-ingestion. Upon leaving, parents were instructed to observe the participants at-home for the next 4 hours and report any adverse events within 24-hours post-ingestion. At baseline and at one year, participants underwent a blood draw. Allergen-specific IgE and IgG4 were measured using a standard ImmunoCAP assay (Phadia, Uppsala, Sweden). Oral food challenges (up to $8 \mathrm{~g}$ of total protein from the 10-food allergen mixture) were conducted between 2-4 years after the start of the study in a facility with trained personnel with staged, monitored standard methods. Questionnaires were provided at baseline and one year and at the time of food challenge. 


\section{Statistical Analysis}

The changes of allergen-specific IgE, IgG4, and IgG4 to IgE ratio from baseline to 1 year are depicted as box plots. The Wilcoxon signed rank test was performed to determine whether the change was significant from baseline to 1 year. The Benjamini-Hochberg $(\mathrm{BH})$ procedure was used to control False Discovery Rate (FDR) for multiple comparisons among all multi-allergen groups for each marker. Q value was used to denote adjusted $\mathrm{p}$ value. Food challenge outcome at 1 year was illustrated using a bar chart, success rate of participants passing food challenge between groups were compared using chi-square test. $\mathrm{P}$ values were adjusted using $\mathrm{BH}$ procedures and denoted as Q values. All tests were two-sided and conducted at the 0.05 level of significance. All analyses were conducted using R software v4.0.3. Descriptive statistics for questionnaire results and demographic information were documented in tables. 


\section{RESULTS}

One hundred and eighty healthy infants aged 2 months to 12 months were recruited at a single site. In all arms, participants were randomized and stratified so that $50 \%$ of participants were at high risk for FA, and 50\% of participants were not at risk of FA. Each of the active groups were further randomized into specific allergen groups as depicted in the consort diagram (Figure 1). No participant dropped out early from the study. Infants consumed daily proteins for an average of $12.1+/-3.2$ months. There was a $95 \%$ adherence rate for each infant. The control group avoided the same 10 food proteins for the first 12 months. All children were breastfed to at least 6 months of age. All participants had available values (or there were no missing values found) for all tested allergen-specific IgE, IgG4, and food challenge outcomes.

Patient demographics are shown in Table 1. In this cohort, $149(83 \%)$ reported having atopic dermatitis. $51 \%$ were of high risk and this was evenly spread throughout all the cohorts. $51 \%$ were female. Median age at start of study was 6 months. Eleven percent were Hispanic, 20\% were Asian, 51\% Caucasian, 14\% African American, and 4\% Pacific Islander.

For safety parameters, across all active groups, there were no increases in allergic reactions reported in participants regardless of risk stratification and eczema co morbid condition. Reactions, all mild skin rashes, were reported in both the control group, $n=4$ (9\%), and active groups, $n=11(8 \%)$. None of those with the mild skin rashes had evidence of eczema or documented family history. Eczema (mild, moderate, and severe) decreased in both controls and in all active groups (Table 2) over time. 
Figure 2 indicates the results of oral food challenges (OFCs). The percent of participants able to consume $8 \mathrm{~g}$ of protein was significantly higher in all mixed protein groups compared to controls $(\mathrm{q}<0.01)$. Interestingly, results from questionnaires demonstrated that those on the mixture diet were more apt to diversify the diet of their infant compared to single, double, and control groups. (Supplementary Table 1)

Specific IgG4, sIgE and IgG4/IgE ratios for all 10 allergens are shown in Figure 3a-3j. The $\mathrm{IgG} 4 / \mathrm{IgE}$ ratios for all mixed protein groups (low, medium, and high) were significantly higher at end of study compared to baseline $(\mathrm{q}<0.1)$, even for peanut, egg, or milk compared to single early intervention active arms. The increase in the ratio can be mainly attributed to increases in IgG4 concentration rather than decreases in IgE. Peanut, milk, and egg biomarkers show improvement compared to control however specificity was demonstrated only with peanut, milk, and egg containing food, respectively. 


\section{Discussion and Conclusion}

While a number of studies have established the safety and efficacy of early introduction of single allergens in infants for the prevention of FA, few studies have evaluated the dose, timing, and number of allergens that can be safely introduced in infants particularly in those with eczema or those at high risk of atopy.(26) We therefore conducted a study to determine the safety and efficacy of early introduction of single, double, and multiple allergens in infants for the prevention of FAs.

Our data suggest that consumption of single, double, and multiple allergens daily in infants is safe at up to $3000 \mathrm{mgs}$ of protein. No increases in the incidence of eczema were observed in infants in the control or active groups, even in infants who had eczema at enrollment or those at high risk of atopy. The safety of multiple allergen introduction has been supported by a few other studies. The Holl study fed infants up to 16 allergens simultaneously with no adverse events. However, it should be noted that in the Holl study, the total protein amount in the allergen mix was only $480 \mathrm{mg}$, an amount much lower than the highest amount used in our study. Further, the study only recruited healthy infants with no severe eczema. The EAT study, which also concluded that early introduction of multiple allergens in infants was safe, differed from our study in that multiple allergens were introduced sequentially rather than simultaneously and that infants were recruited from the general population. The amount used in the EAT study (2000 mgs) was also lower than that used in our study (3000 mgs). The study by Cox et al. found infrequent reactions $(0.1 \%)$ to early introduction of allergenic products similar or lower to what would be expected in a typical US infant population, indicating that early introduction with low doses is within safety parameters for typical feeding. (27) 
Food challenge and biomarker data from our study also suggest that a daily dose of multiple allergens is efficacious for the prevention of FA, even at low amounts (total $300 \mathrm{mg}$ or $30 \mathrm{mg}$ of each protein). This amount was found to be sufficient for decreasing positive food challenge responses and increasing specific food allergen IgG4/IgE. Our study found no difference in sIgE levels for all ten allergens between baseline and 1 year in the active groups. However, sIgG4/sIgE ratio for all 10 allergens were significantly increased for mixtures (low, medium, and high) at the 1-year time point compared to baseline. The change in the sIgG4/sIgE ratio was due to increases in IgG4 rather than decreases in sIgE. The results of this study are similar to other studies, which have shown sIgE and sIgG4 are indicative of allergy and tolerance, respectively. In the LEAP study, peanut-specific IgG4 increased in the peanut consumption group while a greater percentage of participants in the avoidance group had elevated titers of peanut-specific IgE.(11). A large randomized trial of egg introduction from 4 months of age in infants at risk for egg allergy found that levels of IgG4 to egg proteins and IgG4/IgE ratios were higher in those randomized to egg than in controls at 12 month; however, there was no significant difference in egg-specific sIgE levels at 12 months (28). Numerous immunotherapy studies in children for FA have also found increased ratio of sIgG4/sIgE to be indicative of tolerance.(29-34). Overall, our results demonstrate superior efficacy in food challenge outcomes in those individuals fed with the multiple mixture vs. single vs. double as early introduction. A possible reason why the efficacy data shown in our study for multiple allergens is improved over single or double food early intervention could be due to a synchronous effect in inducing tolerance. It has been hypothesized that consumption of multiple allergens simultaneously increases polyclonal $\mathrm{T}$ cell memory subsets, increasing Th1 receptor 
diversity, leading to enhanced protection against potentially allergenic proteins compared to double or single food early intervention.(35)

Our study had lower dropout rates and safety issues compared to other multifood introduction studies (18). We believe regular daily dosing of small amounts of mixed food proteins was key to the success of this study. Participants reported that remembering to eat the powder mixture daily in small amounts was easier than if they were to dose 2-3 times a week with a larger serving of many foods. Further, in the EAT study, the mixture of allergens was prepared at patients' homes; individually prepared allergen mixes could be associated with dosing/serving size variations. Use of premixed allergens as used in this study would reduce this variability. Lastly, families who had taken the mixture of foods as early intervention felt more comfortable having their children eat table foods.

The results of this study suggest that infants 2 months or older, even those with eczema or those at high risk of atopy, can safely and conveniently consume small amounts of multiple allergens daily for the prevention of food allergies. Although $3000 \mathrm{mg}$ of allergenic protein was found to be safe, this high amount is impractical and can lead to noncompliance(19). It would not impede optimal nutrient intake in the infant per USDA feeding guidelines for infants. The low mixture amount (300 mg protein) used in our study does not add significant calories and is not a substitute for foods but instead the clinical data here shows that it can be easily used with breast feeding and/or table foods when the child was ready.

Author Contributions: ZAQ, TAL, and VS wrote article; RD and KGJ wrote article and drew figures; MW manufactured the (allergenic) substance; AT developed the process for making the salmon and shrimp powders used in the allergen mixtures; RSC and SS conducted the study, SC 
created the statistical analysis plan, performed the analysis, reviewed, and revised the manuscript, and $\mathrm{KCN}$ wrote article.

Funding: Sean N. Parker Center for Allergy and Asthma Research at Stanford University.

Institutional Review Board Statement: The study was approved by Stanford IRB.

Informed Consent Statement: We obtained informed consent from parents or caregivers of all infants enrolled in the study.

Patents: The following patent is associated with this work: "Special Oral Formula for Decreasing Food Allergy Risk and Treatment for Food Allergy."

\section{Data Availability Statement:}

The data presented in this study are available on request from the corresponding author.

Conflicts of Interest: Dr. Sindher reports grants from NIH, Regeneron, DBV, AIMMUNE, Novartis, CoFAR, and FARE. She receives personal fees from Astra Zeneca, DBV, and honoraria from FARE. Dr. Chinthrajah reports grants from NIAID, CoFAR, Aimmune, DBV Technologies, Astellas, Regeneron, Stanford Maternal and Child Health Research Institute (MCHRI), and FARE. She is an Advisory Board Member at Alladapt Therapeutics, Novartis, Genentech, Sanofi, Allergenis, and Nutricia. Dr. Nadeau reports grants from National Institute of Allergy and Infectious Diseases (NIAID), National Heart, Lung, and Blood Institute (NHLBI), 
National Institute of Environmental Health Sciences (NIEHS), and Food Allergy Research \& Education (FARE); stock options from IgGenix, Seed Health, ClostraBio, and ImmuneID; is Director of World Allergy Organization (WAO), Advisor at Cour Pharma, Consultant for Excellergy, Red tree ventures, Eli Lilly, and Phylaxis, Co-founder of Before Brands, Alladapt, Latitude, and IgGenix; and National Scientific Committee member at Immune Tolerance Network (ITN), and National Institutes of Health (NIH) clinical research centers, outside the submitted work; patents include, "Mixed allergen composition and methods for using the same", "Granulocyte-based methods for detecting and monitoring immune system disorders", "Methods and Assays for Detecting and Quantifying Pure Subpopulations of White Blood Cells in Immune System Disorders," and "Methods of isolating allergen-specific antibodies from humans and uses thereof." Dr. Tetteh is currently employed by BASF corporation. All other authors indicate no Conflict of Interest. 


\section{REFERENCES}

1. Allen KJ, Koplin JJ. The epidemiology of IgE-mediated food allergy and anaphylaxis. Immunol Allergy Clin North Am. 2012;32(1):35-50.

2. Jackson KD, Howie LD, Akinbami LJ. Trends in allergic conditions among children: United States, 1997-2011. NCHS Data Brief. 2013(121):1-8.

3. Tang ML, Mullins RJ. Food allergy: is prevalence increasing? Intern Med J. 2017;47(3):256-61.

4. Gupta RS, Warren CM, Smith BM, Jiang J, Blumenstock JA, Davis MM, et al. Prevalence and Severity of Food Allergies Among US Adults. JAMA Netw Open. 2019;2(1):e185630.

5. Gupta RS, Warren CM, Smith BM, Blumenstock JA, Jiang J, Davis MM, et al. The Public Health Impact of Parent-Reported Childhood Food Allergies in the United States. Pediatrics. 2018;142(6).

6. Greenhawt M. Food allergy quality of life and living with food allergy. Curr Opin Allergy Clin Immunol. 2016;16(3):284-90.

7. Bergmann MM, Caubet JC, Boguniewicz M, Eigenmann PA. Evaluation of food allergy in patients with atopic dermatitis. J Allergy Clin Immunol Pract. 2013;1(1):22-8.

8. Cox A, Sicherer SH. Peanut and tree nut allergy. Chem Immunol Allergy. 2015;101:131-

44.

9. Ebisawa M, Ito K, Fujisawa T, Committee for Japanese Pediatric Guideline for Food Allergy TJSoPA, Clinical Immunology TJSoA. Japanese guidelines for food allergy 2017.

Allergol Int. 2017;66(2):248-64. 
10. Zeiger RS. Food allergen avoidance in the prevention of food allergy in infants and children. Pediatrics. 2003;111(6 Pt 3):1662-71.

11. Du Toit G, Roberts G, Sayre PH, Bahnson HT, Radulovic S, Santos AF, et al. Randomized trial of peanut consumption in infants at risk for peanut allergy. N Engl J Med. 2015;372(9):803-13.

12. Ierodiakonou D, Garcia-Larsen V, Logan A, Groome A, Cunha S, Chivinge J, et al. Timing of Allergenic Food Introduction to the Infant Diet and Risk of Allergic or Autoimmune Disease: A Systematic Review and Meta-analysis. JAMA. 2016;316(11):1181-92.

13. Roduit C, Frei R, Depner M, Schaub B, Loss G, Genuneit J, et al. Increased food diversity in the first year of life is inversely associated with allergic diseases. J Allergy Clin Immunol. 2014;133(4):1056-64.

14. Venter C, Maslin K, Holloway JW, Silveira LJ, Fleischer DM, Dean T, et al. Different Measures of Diet Diversity During Infancy and the Association with Childhood Food Allergy in a UK Birth Cohort Study. J Allergy Clin Immunol Pract. 2020;8(6):2017-26.

15. Venter C, Greenhawt M, Meyer RW, Agostoni C, Reese I, du Toit G, et al. EAACI position paper on diet diversity in pregnancy, infancy and childhood: Novel concepts and implications for studies in allergy and asthma. Allergy. 2020;75(3):497-523.

16. Koplin JJ, Allen KJ, Tang MLK. Important risk factors for the development of food allergy and potential options for prevention. Expert Rev Clin Immunol. 2019;15(2):147-52. 17. du Toit G, Sayre PH, Roberts G, Lawson K, Sever ML, Bahnson HT, et al. Allergen specificity of early peanut consumption and effect on development of allergic disease in the Learning Early About Peanut Allergy study cohort. J Allergy Clin Immunol. 2018;141(4):134353. 
18. Perkin MR, Logan K, Tseng A, Raji B, Ayis S, Peacock J, et al. Randomized Trial of Introduction of Allergenic Foods in Breast-Fed Infants. N Engl J Med. 2016;374(18):1733-43.

19. USDA. Infant Nutrition and Feeding 2019 [Available from:

https://wicworks.fns.usda.gov/sites/default/files/media/document/Infant_Nutrition_and_Feeding

_Guide.pdf.

20. Holl JL, Bilaver LA, Finn DJ, Savio K. A randomized trial of the acceptability of a daily multi-allergen food supplement for infants. Pediatr Allergy Immunol. 2020;31(4):418-20.

21. Ashley S, Dang T, Koplin J, Martino D, Prescott S. Food for thought: progress in understanding the causes and mechanisms of food allergy. Curr Opin Allergy Clin Immunol. 2015;15(3):237-42.

22. Halken S, Muraro A, de Silva D, Khaleva E, Angier E, Arasi S, et al. EAACI guideline: Preventing the development of food allergy in infants and young children (2020 update). Pediatr Allergy Immunol. 2021;32(5):843-58.

23. Fleischer DM, Chan ES, Venter C, Spergel JM, Abrams EM, Stukus D, et al. A Consensus Approach to the Primary Prevention of Food Allergy Through Nutrition: Guidance from the American Academy of Allergy, Asthma, and Immunology; American College of Allergy, Asthma, and Immunology; and the Canadian Society for Allergy and Clinical Immunology. J Allergy Clin Immunol Pract. 2021;9(1):22-43 e4.

24. Greer FR, Sicherer SH, Burks AW, Committee On N, Section On A, Immunology. The Effects of Early Nutritional Interventions on the Development of Atopic Disease in Infants and Children: The Role of Maternal Dietary Restriction, Breastfeeding, Hydrolyzed Formulas, and Timing of Introduction of Allergenic Complementary Foods. Pediatrics. 2019;143(4). 
25. Koplin JJ, Allen KJ, Gurrin LC, Peters RL, Lowe AJ, Tang ML, et al. The impact of family history of allergy on risk of food allergy: a population-based study of infants. Int $\mathbf{J}$ Environ Res Public Health. 2013;10(11):5364-77.

26. Schroer B, Groetch M, Mack DP, Venter C. Practical Challenges and Considerations for Early Introduction of Potential Food Allergens for Prevention of Food Allergy. J Allergy Clin Immunol Pract. 2021;9(1):44-56 e1.

27. Cox AL, Shah A, Groetch M, Sicherer SH. Allergic reactions in infants using commercial early allergen introduction products. J Allergy Clin Immunol Pract. 2021;9(9):3517-20 e1.

28. Wei-Liang Tan J, Valerio C, Barnes EH, Turner PJ, Van Asperen PA, Kakakios AM, et al. A randomized trial of egg introduction from 4 months of age in infants at risk for egg allergy. J Allergy Clin Immunol. 2017;139(5):1621-8 e8.

29. Kulis M, Yue X, Guo R, Zhang H, Orgel K, Ye P, et al. High- and low-dose oral immunotherapy similarly suppress pro-allergic cytokines and basophil activation in young children. Clin Exp Allergy. 2019;49(2):180-9.

30. Santos AF, Brough HA. Making the Most of In Vitro Tests to Diagnose Food Allergy. J Allergy Clin Immunol Pract. 2017;5(2):237-48.

31. Jones SM, Pons L, Roberts JL, Scurlock AM, Perry TT, Kulis M, et al. Clinical efficacy and immune regulation with peanut oral immunotherapy. J Allergy Clin Immunol.

2009;124(2):292-300, .e1-97.

32. Vickery BP, Lin J, Kulis M, Fu Z, Steele PH, Jones SM, et al. Peanut oral immunotherapy modifies IgE and IgG 4 responses to major peanut allergens. J Allergy Clin Immunol. 2013;131(1):128-34. e3. 
33. Varshney P, Jones SM, Scurlock AM, Perry TT, Kemper A, Steele P, et al. A randomized controlled study of peanut oral immunotherapy: clinical desensitization and modulation of the allergic response. J Allergy Clin Immunol. 2011;127(3):654-60.

34. Chinthrajah RS, Purington N, Andorf S, Long A, O'Laughlin KL, Lyu SC, et al. Sustained outcomes in oral immunotherapy for peanut allergy (POISED study): a large, randomised, double-blind, placebo-controlled, phase 2 study. Lancet. 2019;394(10207):1437-49. 35. Sampath V, Nadeau KC. Newly identified T cell subsets in mechanistic studies of food immunotherapy. J Clin Invest. 2019;129(4):1431-40. 
Tables and Figures

\section{Table 1}

\begin{tabular}{|l|l|}
\hline & $\mathrm{N}=180$ \\
\hline Female Sex & $91(51 \%)$ \\
\hline $\begin{array}{l}\text { Ethnicity } \\
\text { - Asispanic }\end{array}$ & $20(11 \%)$ \\
- Caucasian & $36(20 \%)$ \\
- Afro American & $91(51 \%)$ \\
- Pacific Islander & $25(14 \%)$ \\
\hline Age & $8(4 \%)$ \\
\hline Eczema (via Scorad) & $149(83 \%)$ \\
Mild & $83(46 \%)$ \\
Moderate & $45(25 \%)$ \\
Severe & $21(12 \%)$ \\
\hline High Risk* & $\mathrm{N}=91(51 \%)$ \\
Family hx & \\
\hline
\end{tabular}

*: High risk was defined as one first degree relative with food allergy/atopic dermatitis or two first degree relatives with atopic disease. 
Figure 2: Safety: Adverse reactions during study

\begin{tabular}{|l|l|l|l|l|}
\hline & $\begin{array}{l}\text { Control N=45 at } \\
\text { baseline }\end{array}$ & $\begin{array}{l}\text { Control arm } \\
\text { reactions during } \\
\text { study }\end{array}$ & $\begin{array}{l}\text { Active N=135 at } \\
\text { baseline }\end{array}$ & $\begin{array}{l}\text { Active arm } \\
\text { reactions during } \\
\text { study }\end{array}$ \\
\hline No eczema & $8(18 \%)$ & $\begin{array}{l}4(\text { all mild rash) } \\
(9 \%)\end{array}$ & $22(16 \%)$ & $\begin{array}{l}11(\text { all mild } \\
\text { rash) }(8 \%)\end{array}$ \\
\hline Mild eczema & $22(49 \%)$ & $1(2 \%)$ & $61(45 \%)$ & $2(1 \%)$ \\
\hline $\begin{array}{l}\text { Moderate } \\
\text { eczema }\end{array}$ & $10(22 \%)$ & none & $35(26 \%)$ & $4(3 \%)$ \\
\hline Severe eczema & $5(11 \%)$ & $1(2 \%)$ & $17(13 \%)$ & none \\
\hline $\begin{array}{l}\text { High risk* } \\
\text { Family hx }\end{array}$ & $\mathrm{N}=23(51 \%)$ & $1(2 \%)$ & $\mathrm{N}=69(51 \%)$ & $3(2 \%)$ \\
\hline
\end{tabular}


Figure 1: consort diagram

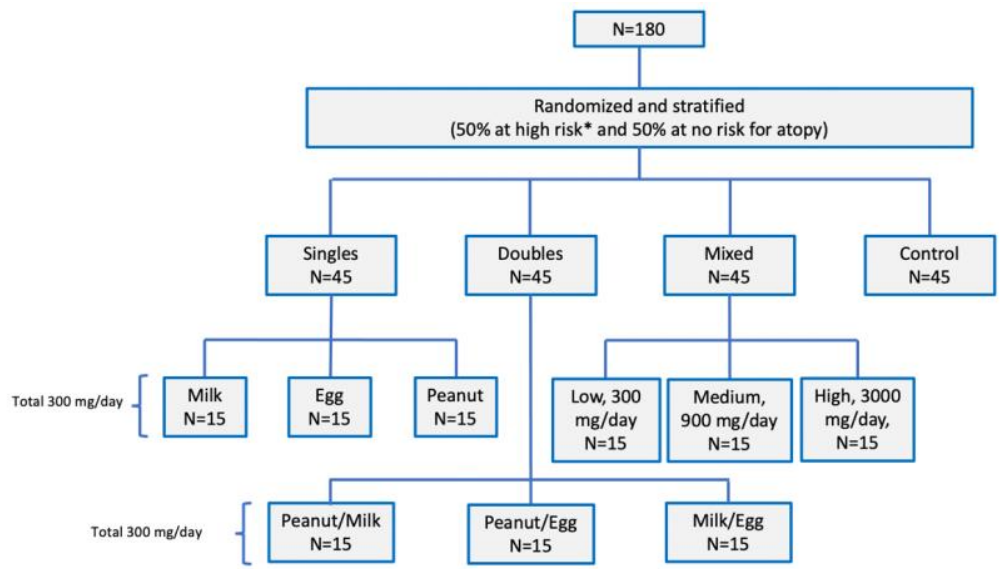

*: High risk was defined as one first degree relative with food allergy/atopic dermatitis or two first degree relatives with atopic disease 
Figure 2: Oral Food Challenges

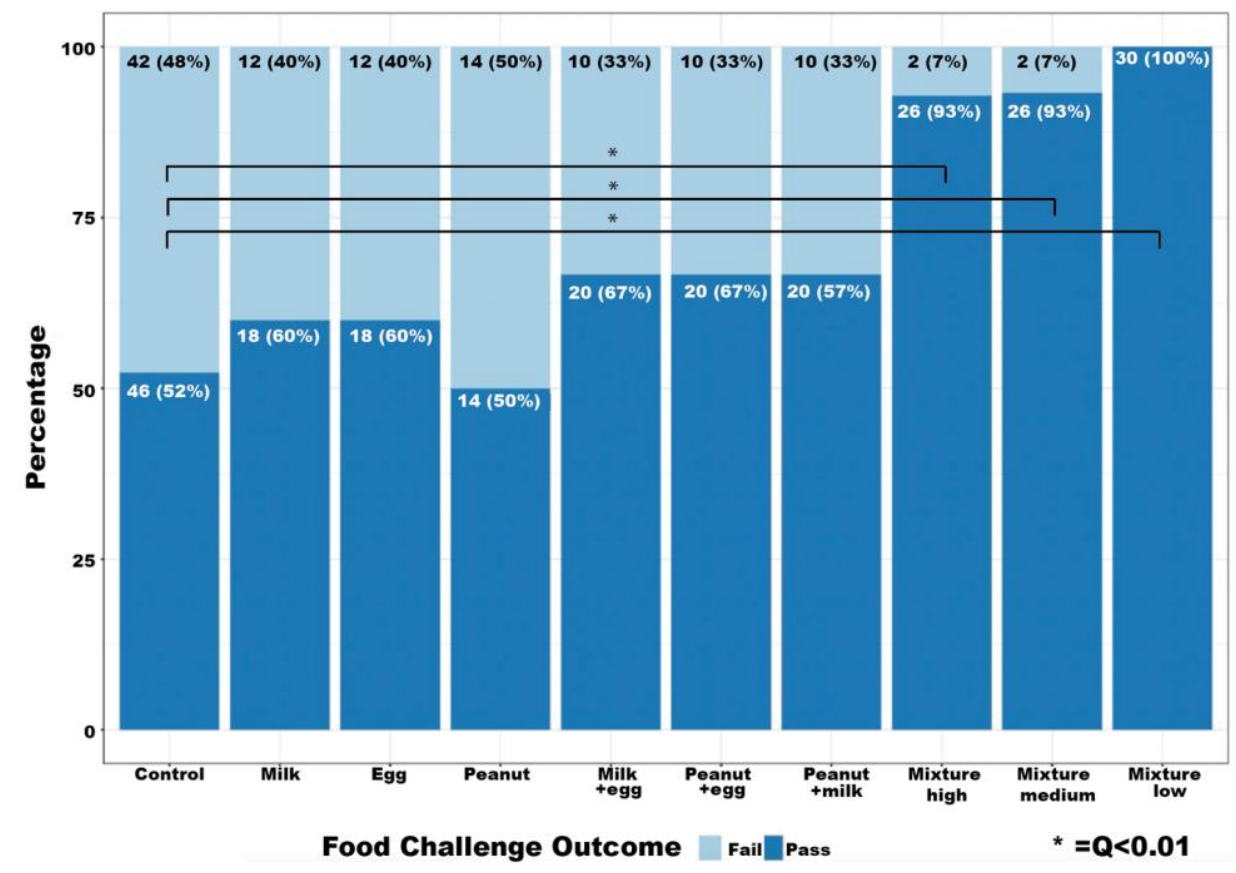


Figure 3: IgE and IgG4 and Food Challenge Outcome

A. Almond
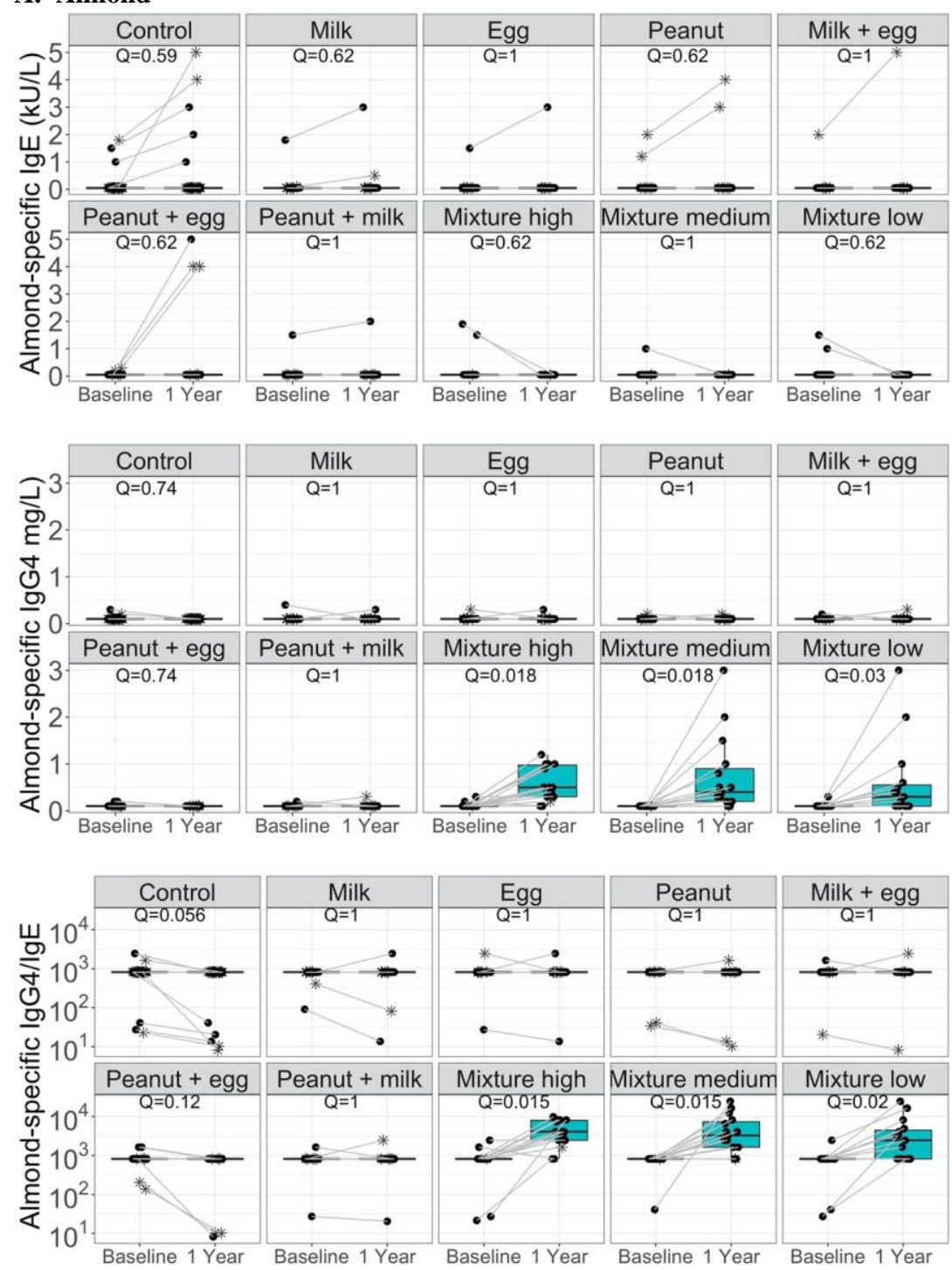

Study time Baseline 1 Year Food challenge outcome * Fail - Pass 
B. Cashew
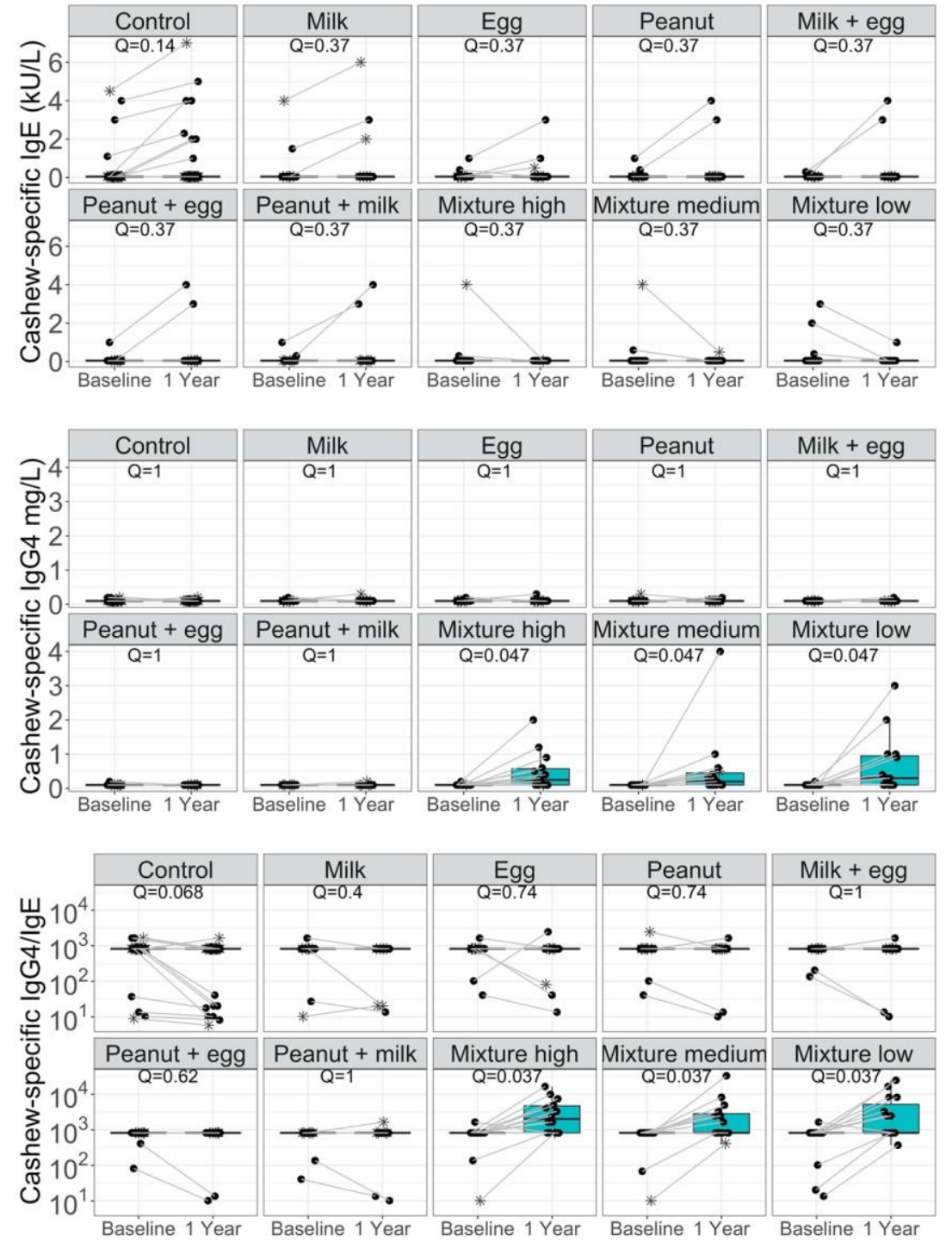

Study time Baseline 1 Year Food challenge outcome * Fail - Pass 


\section{Egg}
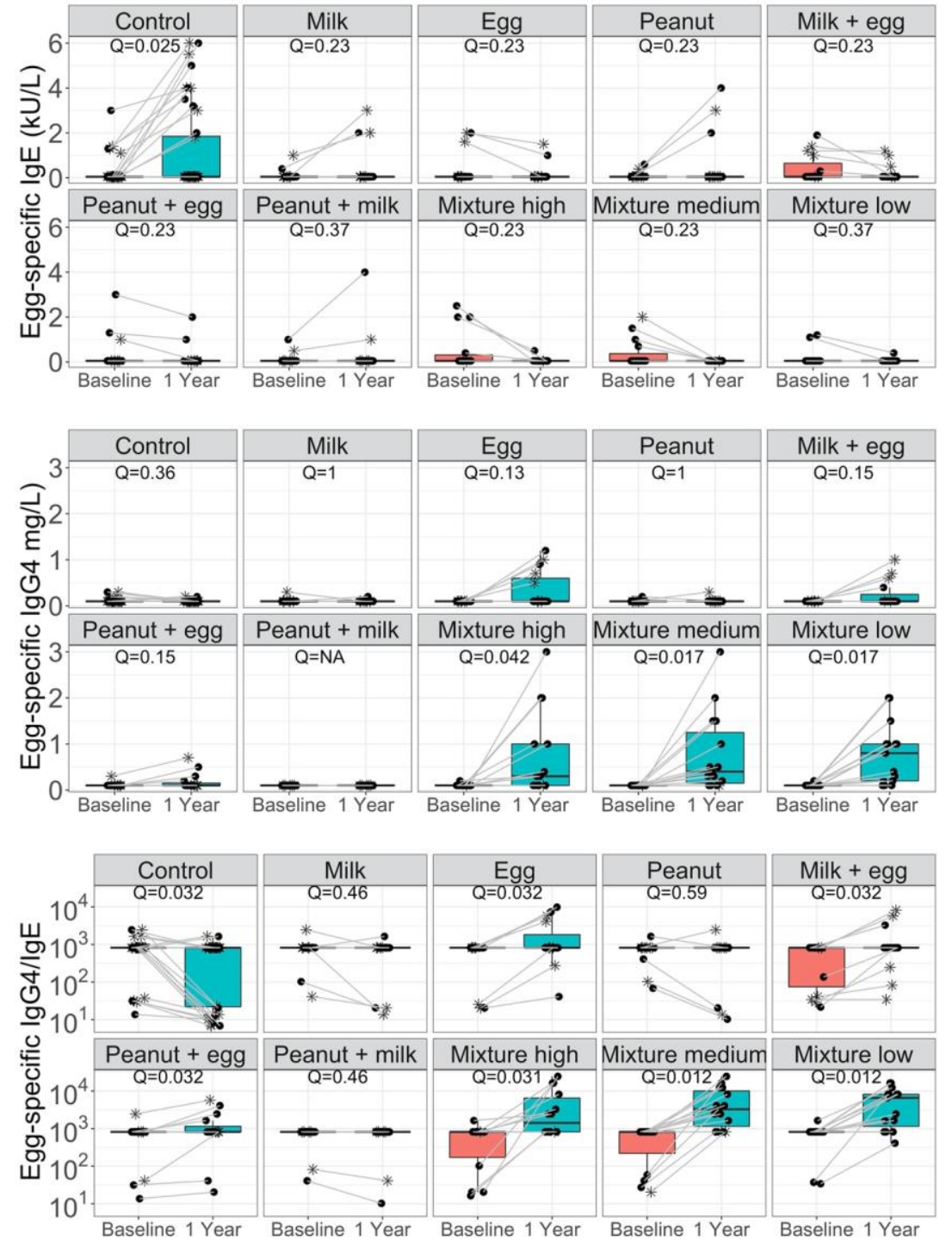

Study time Baseline 1 Year Food challenge outcome* Fail Pass 


\section{Hazelnut}
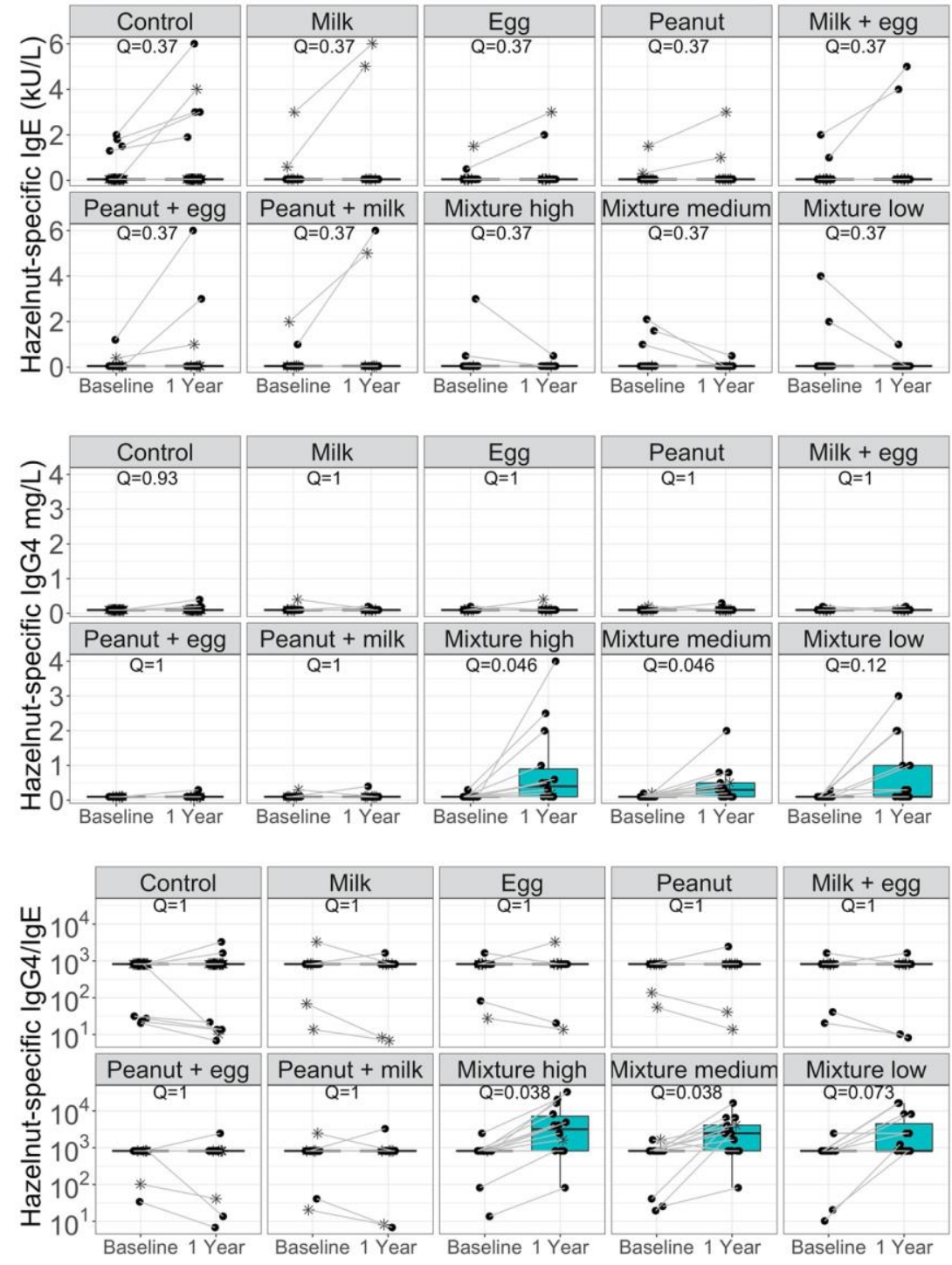

Study time Baseline 1 Year Food challenge outcome* Fail Pass 
E. Milk
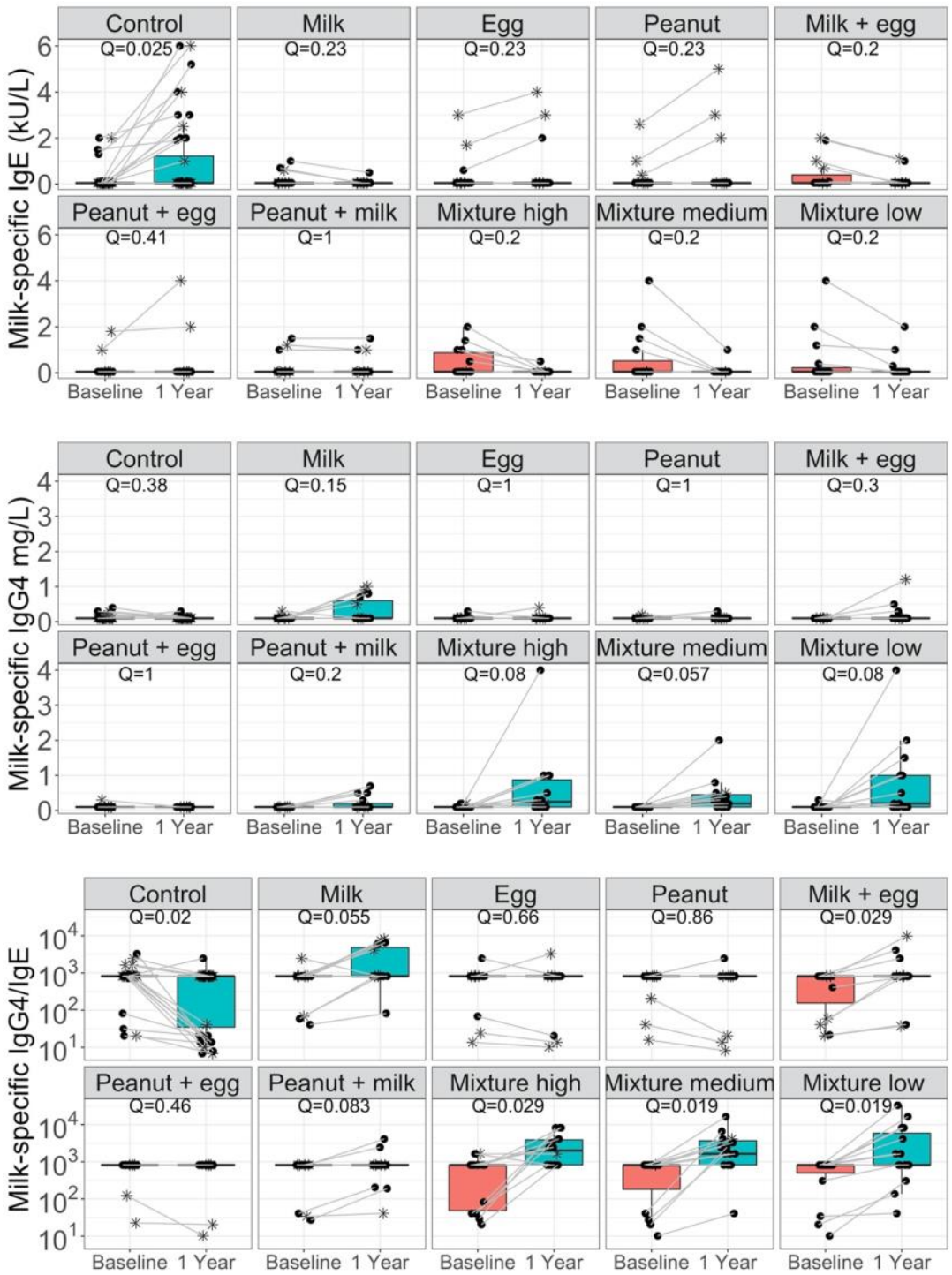

Study time Baseline 1 Year Food challenge outcome * Fail Pass 


\section{F. Peanut}
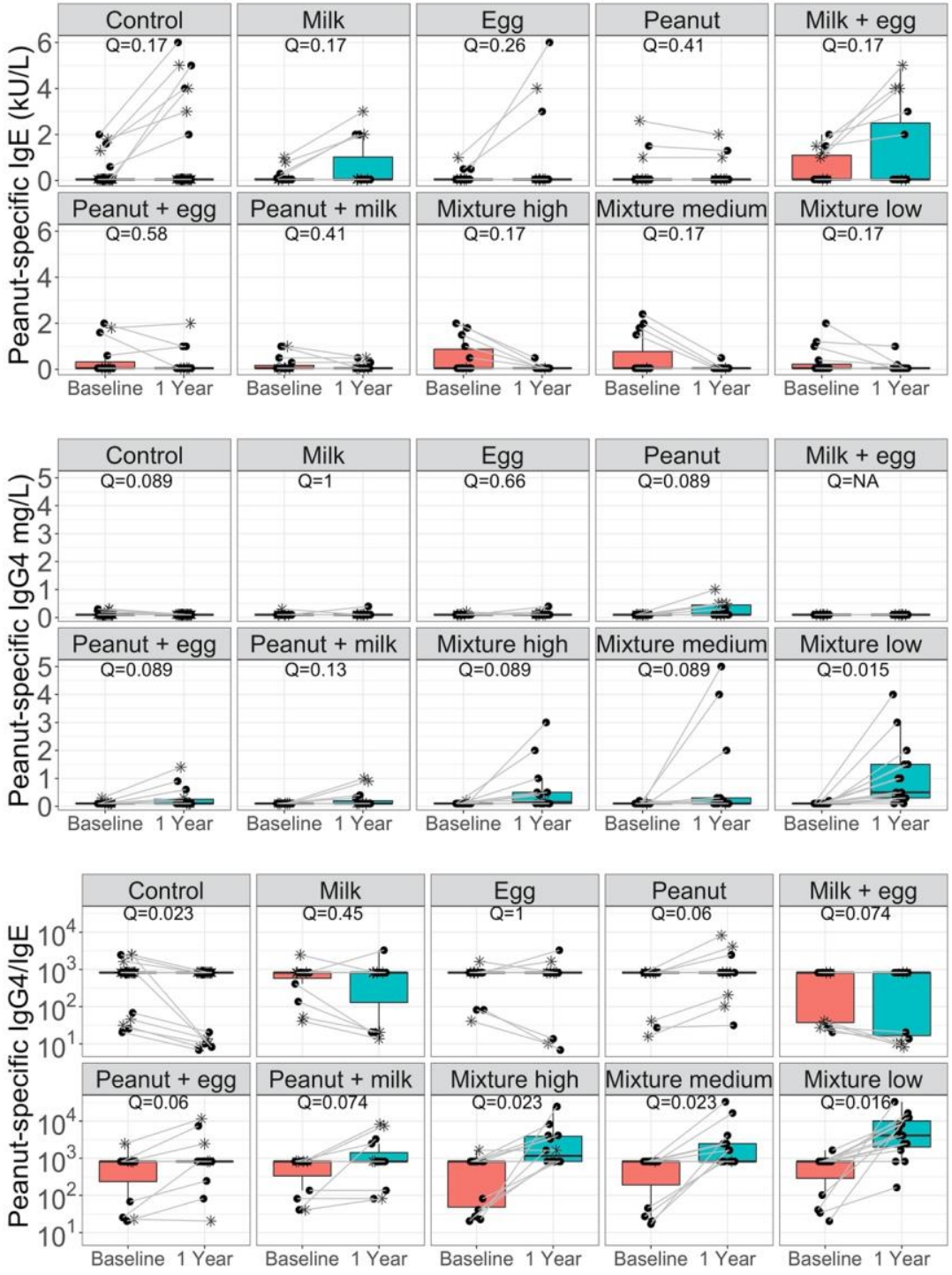

Study time Baseline 1 Year Food challenge outcome * Fail - Pass 


\section{G. Salmon}
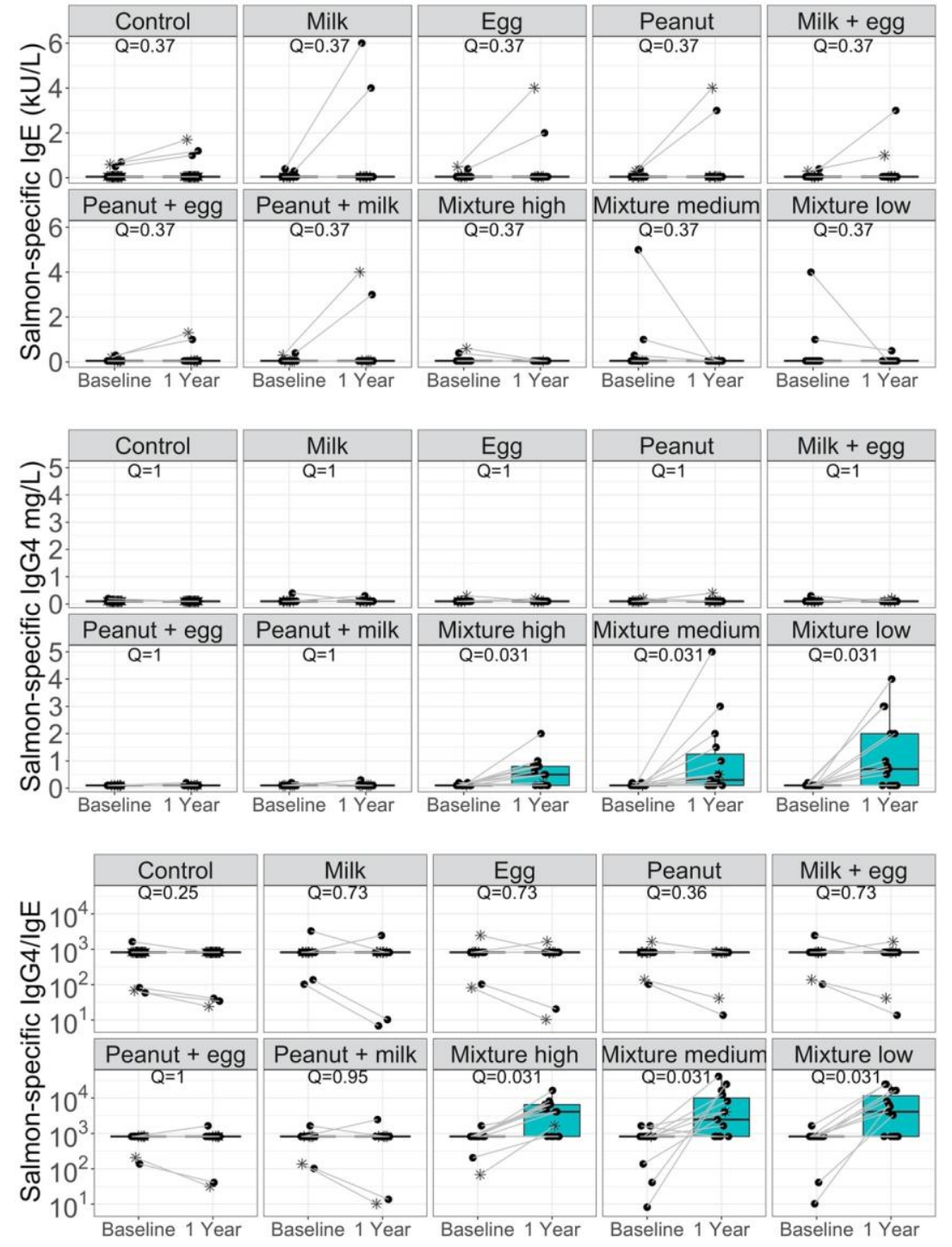

Study time Baseline 1 Year Food challenge outcome* Fail Pass 


\section{H. Shrimp}
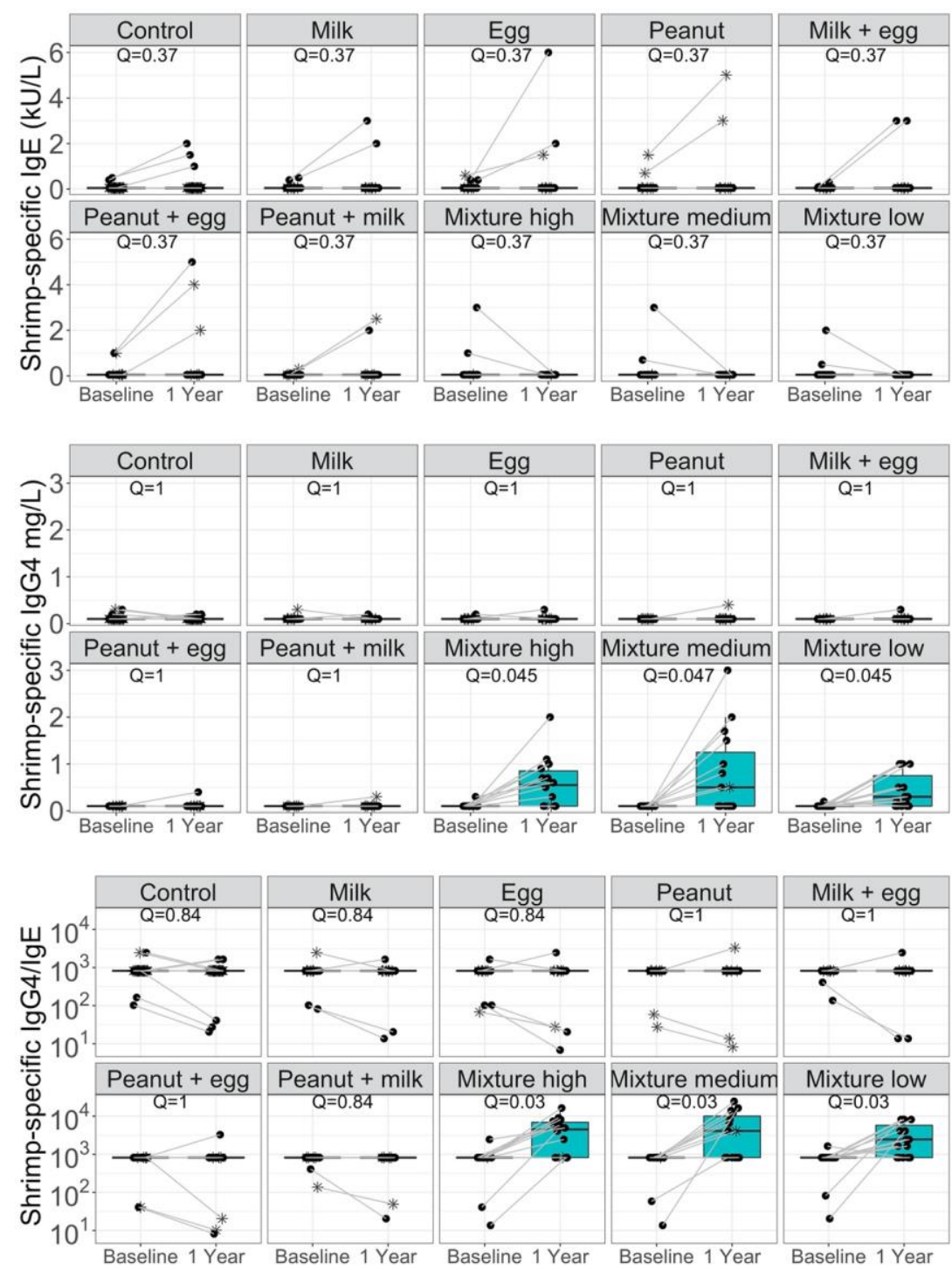

Study time Baseline 1 Year Food challenge outcome* Fail Pass 


\section{Walnut}
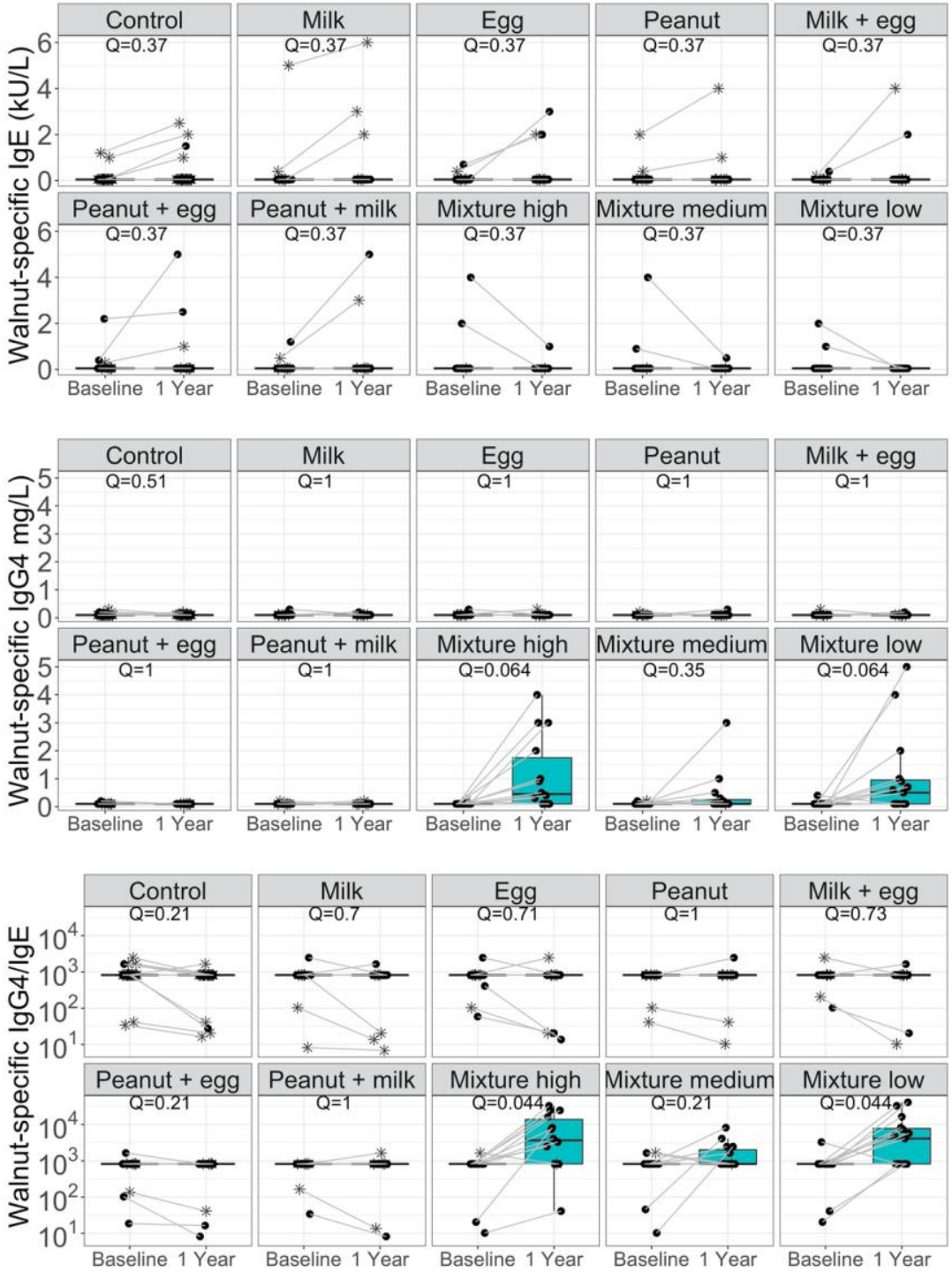

Study time Baseline 1 Year Food challenge outcome* Fail Pass 


\section{J. Wheat}
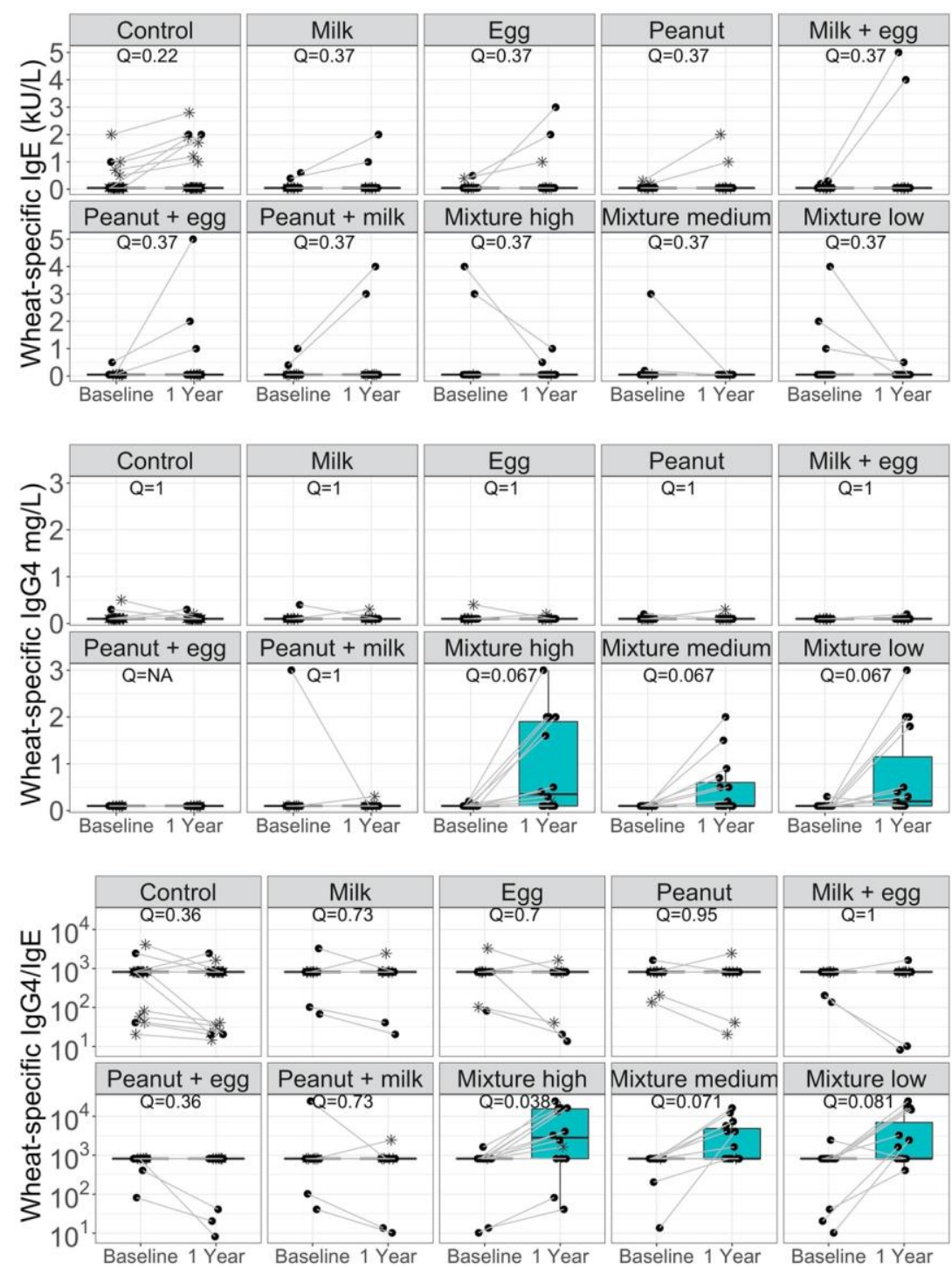

Study time Baseline 1 Year Food challenge outcome* Fail Pass 
Supplementary Table 1: Number of infants fed more than 10 foods by 1 year of age

\begin{tabular}{|l|l|}
\hline Allergen group & $\mathrm{N}$ \\
\hline Mixture (low dose) & 15 \\
\hline Mixture (medium dose) & 14 \\
\hline Mixture (high dose) & 15 \\
\hline Control & 7 \\
\hline Peanut & 10 \\
\hline Milk & 8 \\
\hline Egg & 9 \\
\hline Peanut/milk & 11 \\
\hline Milk/egg & 10 \\
\hline Egg/peanut & 8 \\
\hline
\end{tabular}

